\title{
TRATAMIENTO BIOLÓGICO DE AGUAS RESIDUALES DE MATADERO. CASO: FRIGORÍFICO LA FRONTERA LTDA., VILLA DEL ROSARIO, NORTE DE SANTANDER
}

Por:

Néstor Andrés Urbina Suárez', Sandra Liliana Pabón², John Hermógenes Suárez Gélvez ${ }^{3}$

\section{RESUMEN}

La investigación realizada caracterizó y cuantificó el agua residual vertida por el Frigorífico "La Frontera Ltda." del municipio de Villa del Rosario N.S. y evaluó y operó un sistema de tratamiento Biológico a escala industrial con la infraestructura física disponible en el matadero, con el objeto de evaluar la factibilidad técnica para diseñar una planta a escala real.

La mayor producción de agua residual en el matadero durante el tiempo de estudio, se presentó en los meses de mayo con un caudal de $3.93 \mathrm{l} / \mathrm{s}$ y diciembre con un caudal de $3.37 \mathrm{l} / \mathrm{s}$, correspondiendo con las épocas de mayor sacrifico de reses. La menor producción de agua residual se registró en los meses de agosto con un caudal $2.49 \mathrm{l} / \mathrm{s}$ y marzo con un caudal de $2.61 \mathrm{l} / \mathrm{s}$. La DQO del agua residual se encontraba entre 14150 y $16900 \mathrm{mg} / \mathrm{l}$, y la DBO varió entre 6950 y 7980 mg/l.

Para la construcción de los reactores de lodos activos, se adecuaron tanques circulares en acero inoxidable con una capacidad de $8 \mathrm{~m}^{3}$. Cada reactor trabajó con inóculos diferentes: el reactor 1 con un inóculo activado a partir de rumen del sedimentador primario, el reactor 2 con un inóculo activado a partir del rumen retenido por las cribas y el reactor 3 con un inóculo comercial. La remoción promedio en DQO y DBO fueron: $78.12 \%$ en DQO y $85.75 \%$ en $\mathrm{DBO}$ para el reactor $1,78.65 \%$ en DQO y $72.12 \%$ DBO para el reactor 2 y $64.92 \%$ en DQO y $56.44 \%$ en DBO para el reactor 3.

Durante la operación de los reactores se aislaron e identificaron microorganismos presentes en el lodo activo del reactor 1 que fue el que presentó mejores eficiencias y se identificó la presencia de protozoos en cada reactor.

Palabras clave: Lodos activos, Agua rumen, Agua sangre, $\mathrm{DQO}, \mathrm{DBO}$.

\section{ABSTRACT}

The achieved investigation characterized and quantified the residual water spilt by the The "FRIGORIFICO LA FRONTERA Ltda. " of the municipality of Villa del Rosario Norte de Santander ( Colombia)., it evaluated and operated a system of Biological treatment to industrial scale with the physical available infrastructure in the slaughterhouse, with the purpose of evaluating the technical feasibility in order to design a plant to real scale.

The greatest production of residual water in the slaughterhouse during the time of research in take place in May with a wealth of $3,93 \mathrm{~L} / \mathrm{s}$ and in December with a wealth of $3,37 \mathrm{~L} / \mathrm{s}$, according to the period of highest number of killed animals. The smallest production of residual water was registered in August with a wealth 2,49 L/s and in March with a wealth of $2,61 \mathrm{~L} / \mathrm{s}$. The COD of residual water was situed between 14150 and $16900 \mathrm{mg} / \mathrm{l}$, and the BOD changed between 6950 and $7980 \mathrm{mg} / \mathrm{l}$.

\footnotetext{
'Ingeniero de Producción Biotecnológica, Grupo de Investigación en Biotecnología Ambiental y Aplicada (GIBA), Universidad Francisco de Paula Santander. UFPS. Andres130583@yahoo.es

${ }^{2}$ Ingeniera de Producción Biotecnológica, Grupo de Investigación en Biotecnología Ambiental y Aplicada (GIBA), Universidad Francisco de Paula Santander. UFPS. Sandraliliana64@hotmail.com

${ }^{3}$ M.Sc. en Ingeniería Sanitaria y Ambiental. Director Grupo de Investigación en Biotecnología Ambiental y Aplicada ( GIBA ), Universidad Francisco de Paula Santander, UFPS. johnhermogenes@yahoo.com.
} 
Tratamiento biológico de aguas residuales de matadero. Caso: Frigorífico La Frontera Ltda., Villa del Rosario, Norte de Santander

In the construction of activate sludge reactors, circular tanks on stainless steel were adapted with a capacity of $8 \mathrm{~m} 3$. Each reactor was inoculated of different form: reactor 1 with one inoculated activated from rumen of the primary sedimentador, the reactor 2 with one inoculated activated from the rumen retained by the sieves and the reactor 3 with a commercial inóculo. The removal mediated in DQO and DBO was: $78.12 \%$ in DQO and $85.75 \%$ in DBO for the reactor 1, $78.65 \%$ in DQO and 72.12 $\%$ DBO for the reactor 2 and $64.92 \%$ in DQO and $56.44 \%$ en DBO for the reactor 3 .

During the operation of the reactors present microorganisms isolated and identified in the active mud of the reactor 1 that was the one that presented better efficiencies and the presence of protozoans was identified in every reactor.

Key words: Active muds, It Waters down rumen, blood Waters down, DQO, DBO.

\section{INTRODUCCIÓN}

Las aguas residuales de matadero son aquellas que provienen del proceso de sacrificio y faenado y su composición es el producto de dos fuentes básicas de generación: agua sangre y agua rumen. La primera de ellas se produce en las fases de izado y arreglo de la res en canal, así como durante el lavado de las vísceras rojas. La segunda es aquella que proviene del lavado de las vísceras blancas.

Las aguas residuales de matadero se caracterizan por tener concentraciones altas de DQO y DBO debido a la gran cantidad de grasas que se encuentran en ellas [1]. La variación de estas concentraciones se debe básicamente a la alta producción de agua sangre, cuanta mayor cantidad de producción exista mayor va ser la concentración de carga contaminante en el agua residual. Otros factores que influyen en las características de esta aguas son: cantidad de reses sacrificadas, cantidad de agua utilizada en el proceso, la especie del animal y la dieta que posea, clase y capacidad de las instalaciones del Frigorífico, intensidad de limpieza de las canales, entre otros [2]. El caudal de agua residual generado depende básicamente de la cantidad de reses sacrificadas y la cantidad de agua utilizada en el proceso de sacrificio y faenado.

Los procesos biológicos aeróbicos y anaeróbicos constituyen una forma de resolver el problema del tratamiento de estos vertimientos. Dentro de los tratamientos aerobios se caracterizan las lagunas aireadas y el proceso de lodos activados [3], en lo referente al tratamiento anaerobio se suele utilizar los reactores UASB y las lagunas anaerobias. Dentro de estas dos tecnologías de depuración los lodos activados presentan mayor favorecimiento por los resultados obtenidos en experimentos reportados [4]. En el país el tratamiento de lodos activados es una alternativa de solución como en el matadero de Buga (Valle del Cauca) donde se realizó un sistema de tratamiento de estas características, y en países como Chile y España esta tecnología es una gran alternativa de solución

Antes de proponer cualquier tipo de tecnología, es necesario analizar y estudiar las características de los vertimientos de los mataderos, con el fin de poder elegir en cada caso la alternativa que mejor se adapte a las necesidades.

El Frigorífico "La Frontera Ltda." Se encuentra ubicado en la Carrera 7 calle 25 y 26 Barrio Gran Colombia en el municipio de Villa del Rosario N.S. y presta los servicios de sacrificio y faenado para la demanda de carne en Cúcuta y la Zona Metropolitana.

El caudal de los vertimientos de agua residual del Frigorífico "La Frontera Ltda." se encontraba en un promedio de 3,2 l/s y con una carga de DQO promedio de15232 mg/l y de DBO promedio de 7547 mg/l [5] durante el tiempo de estudio. En el presente trabajo se caracterizaron las aguas residuales y se diseñó y operó un sistema de tratamiento piloto de lodos activados 
Tratamiento biológico de aguas residuales de matadero. Caso: Frigorífico La Frontera Ltda., Villa del Rosario, Norte de Santander

con el fin de evaluar la factibilidad de esta tecnología y generar parámetros que permitieran diseñar una planta de tratamiento final.

\section{MATERIALES Y METODOS}

Caracterización y cuantificación de las aguas residuales del Frigorífico "La Frontera Ltda."

Cuantificación de las aguas residuales: Para conocer el caudal de agua residual generada por el Frigorífico "La Frontera Ltda.", se escogieron dos puntos de monitoreos en donde se cuantificó el caudal de agua residual. El primer punto de muestreo correspondió a la entrada del sedimentador primario (agua rumen) y el segundo punto de muestreo es el de agua sangre, el cual se hizo sobre el vertimiento a uno de los tanques que descarga al alcantarillado. La suma de los caudales de estos dos puntos da como resultado el caudal total.

Para medir la producción de agua residual se realizaron monitoreos periódicos durante los meses de mayo de 2004 a marzo de 2005. La medición de caudal se efectuó utilizando un recipiente aforado de 13 litros de tal forma que se aseguró el tomar un volumen de agua residual relativamente grande y tiempos de llenados no muy cortos, lo cual permitió obtener valores de caudal confiables En cada mes se escogió una semana (lunes - sábado) para monitorear en el horario de la jornada de sacrificio de 2:30pm a 9:30pm, midiendo el caudal de agua residual cada media hora en los dos puntos.

Cuantificación del agua residual generada por etapas. Para medir el caudal de agua residual por etapa de proceso se escogieron tres zonas dentro de la planta para el monitoreo:

- Zona 1: Línea de Faenado.

- Zona 2: Cocina y Sala de Vísceras Rojas

- Zona 3: Sala de Oreo
En cada zona se contó el número de llaves y se midió el caudal de agua que desalojaba abriendo totalmente la llave. Para medir el caudal se aforó un recipiente a 7 litros y se contabilizó el tiempo que se tardó en llenarse este volumen; finalmente se monitoreó cada zona midiendo cuanto tiempo duraba abierta cada una de las llaves por jornada de trabajo.

Caracterización de las aguas residuales: Para el análisis y la caracterización de las aguas residuales del Frigorífico "La Frontera Ltda.", se tomaron muestras compuestas durante las jornadas de faenado, utilizando recipientes con capacidad de 5 galones. Las muestran se tomaron a la salida de la planta de sacrificio. (Antes de cribas)

Los análisis realizados se muestran en la Tabla 1.

Tabla 1. Parámetros y técnicas utilizadas para el análisis de las aguas residuales del frigorífico.

\begin{tabular}{|c|c|}
\hline ANÁLISIS & METODO \\
\hline Demanda química de oxigeno (DQO) & Winkler y Oxitop \\
\hline Demanda bioquímica de oxigeno (DBO) & Colorimetría \\
\hline Nitratos $\left(\mathrm{NO}_{3}\right)$ & Colorimetría \\
\hline Nitritos $\left(\mathrm{NO}_{2}\right)$ & Colorimetría \\
\hline Sulfatos $\left(\mathrm{SO}_{4}\right)$ & Colorimetría \\
\hline Fosfatos $\left(\mathrm{PO}_{4}\right)$ & Colorimetría \\
\hline Alcalinidad & Volumétrico \\
\hline Acidez & Volumétrico \\
\hline Temperatura & Electrométrico \\
\hline PH & Electrométrico \\
\hline Sólidos Sedimentables (SSe) & Volumétrico \\
\hline Sólidos Totales (ST) & Gravimétrico \\
\hline Sólidos suspendidos totales (SST) & Gravimétrico \\
\hline Sólidos suspendidos volátiles (SSV) & Gravimétrico \\
\hline Sólidos suspendidos fijos (SSF) & Gravimétrico \\
\hline Microbiológico & Placa profunda y filtración \\
& por membrana \\
\hline
\end{tabular}

Los análisis se realizaron en el laboratorio de aguas de la Universidad Francisco de Paula Santander (U.F.P.S.), de acuerdo con los protocolos establecidos por el Standard Methods for the Examination of Water and Wastewater APHA (1992). American Public Health Association (APHA), American Water Works Association (AWWA), Water Environment Federation (WEF) [1 1] y los publicados por el IDEAM (disponibles en la pagina Web de este instituto) [12]. 
Tratamiento biológico de aguas residuales de matadero. Caso: Frigorífico La Frontera Ltda., Villa del Rosario, Norte de Santander

Adecuación de la infraestructura disponible en la empresa para el arranque del sistema piloto de lodos activos.

En esta fase del proyecto se analizó que estructuras existentes en el frigorífico podrían ser de utilidad para llevar a cabo el sistema de tratamiento de los efluentes. Estas estructuras fueron utilizadas anteriormente como sistema de tratamiento de agua residual, pero no dieron la capacidad y la eficiencia para tratar el agua.

Se realizó un estudio de cada una de las estructuras tales como cribas, sedimentadores y tanques circulares. Se calcularon las capacidades volumétricas de los tanques para conocer los tiempos de retención hidráulica.

Con el apoyo de la unidad de mantenimiento de la empresa se instaló la conducción y evacuación del agua residual, suministro de energía y se efectuó el sellamiento de los reactores circulares. (Utilizados en el antiguo tratamiento de agua).

Seguimiento y evaluación del sistema de lodos activos.

El tratamiento biológico fue acondicionado con 3 reactores para que operara como un sistema de Lodo activo sin recirculación. Para el control y seguimiento se realizaron seis experimentos con diferentes replicas en donde se analizaron las diferentes variables que afectan el proceso. Las características de cada experimento se muestran en la tabla 2.

Tabla 2. Seguimiento de los reactores de lodos activados por experimento.

\begin{tabular}{|c|c|c|c|c|c|c|c|c|c|c|c|}
\hline \multirow{3}{*}{ EXPERIMENTO } & \multicolumn{11}{|c|}{ VARIABLES } \\
\hline & \multirow{2}{*}{$\begin{array}{c}\mathrm{V} \\
\left(\mathrm{m}^{3}\right)\end{array}$} & \multirow{2}{*}{$\begin{array}{l}\text { QAire } \\
\left(l_{s}\right)\end{array}$} & \multicolumn{3}{|c|}{ [] AR-AS (\%) } & \multicolumn{3}{|c|}{$\theta(\mathrm{h})$} & \multicolumn{3}{|c|}{ Inóculo } \\
\hline & & & R1 & $\mathrm{R} 2$ & R3 & R1 & R2 & R3 & $\overline{\mathrm{R} 1}$ & R2 & R3 \\
\hline 1 & 8 & 6 & $65-35$ & - & - & 36 & - & - & $\mathrm{RS}_{1}$ & . & - \\
\hline 2 & 8 & 6 & $60-40$ & $65-35$ & - & 21 & 36 & & $K S_{1}$ & $\mathrm{RC}_{1}$ &. \\
\hline 3 & 8 & 6 & $50-50$ & $60-40$ & $60-40$ & 18 & 21 & 36 & $\mathrm{RS}_{1}$ & $\mathrm{RC}_{1}$ & Pool \\
\hline 4 & 8 & 6 & $50-50$ & $50-50$ & $50-50$ & 18 & 18 & 21 & $\mathrm{RS}_{1}$ & $\mathrm{RC}_{1}$ & Pool \\
\hline 5 & 8 & 6 & $60-40$ & $60-40$ & - & 21 & 21 & - & $\mathrm{RS}_{2}$ & $\mathrm{RC}_{2}$ & - \\
\hline 6 & 8 & 6 & $50-50$ & $50-50$ & - & 21 & 21 & - & $\mathrm{RS}_{2}$ & $\mathrm{RC}_{2}$ & - \\
\hline
\end{tabular}

Donde:

$R$ 1: Reactor $1 \quad \theta$ : Tiempo de retención AR: Agua rumen

R 2: Reactor $2 \quad$ RS1: Rumen del Sedimentador semilla 1. AS: Agua sangre

R 3: Reactor 3 RS2: Rumen del Sedimentador semilla 2.

Rcl: Rumen de cribas semilla 1 V: Volumen QAire: Caudal del aire

RC2:Rumen de cribas semilla 2 Pool: Inóculo comercial []AR-AS: Concentración

Inóculo: Para llevar a cabo el arranque de la planta piloto fue necesario buscar el lodo adecuado para tratar las aguas residuales. Se analizaron varias opciones y se activaron tres inóculos:

- Un primer inóculo a partir del rumen desalojado por el sedimentador, que se llevó a un lecho de secado eliminando un $60 \%$ de la humedad inicial.

- Un segundo inóculo a partir del rumen desalojado por las cribas, que fue triturado para formar un rumen mas fino y al igual que el inóculo 1 se eliminó el $60 \%$ de humedad.

- El tercer inóculo se obtuvo de un pool comercial que se activó según las especificaciones del fabricante.

A todos los inóculos se les midió los siguientes parámetros SST, SSV y Sedimetabilidad de acuerdo con los protocolos establecidos por el Standard Methods (APHA, AWWA, WEF, 1995) [11] y los publicados por el IDEAM (disponibles en la página Web de este instituto) [12].

Evaluación de la Biología del reactor de lodos activos.

- Aislamiento e identificación de microorganismos del proceso de lodos activos. En esta etapa del proyecto se identificaron los microorganismos que se encuentran en lodo llevando a cabo el proceso de degradación de la materia orgánica. Se tomaron muestras del lodo de cada reactor desde septiembre de 2004 hasta enero de 2005, las cuales se aislaron en una primera instancia y en una segunda instancia se proce- 
Tratamiento biológico de aguas residuales de matadero. Caso: Frigorífico La Frontera Ltda., Villa del Rosario, Norte de Santander

dió a identificar los del reactor 1 que fue el que mayor eficiencia presentó. La identificación se realizó por medio de pruebas bioquímicas.

Identificación de indicadores biológicos.

Como indicadores biológicos sólo se identificaron protozoos por ser los microorganismos más abundantes de la microfauna en los lodos activos, ya que pueden llegar a alcanzar valores medios de 50.000 ind/ $\mathrm{ml}$ en los reactores biológicos constituyendo aproximadamente el $5 \%$ del peso seco de los sólidos en suspensión del licor mezcla, los protozoos además mejoran la calidad del efluente y regulan la biomasa bacteriana al predar sobre las bacterias dispersas del licor de mezcla [6]. Para identificar los protozoos presentes en el lodo se tomaron muestras del lodo y se observaron directamente al microscopio y con la ayuda de textos y referencias bibliográficas [6], [13], [14], se identificaron diversas especies de protozoos.

\section{Caracterización de lodos}

Para la caracterización del lodo y su comportamiento en cada reactor se realizaron pruebas al lodo durante la operación de la planta para determinar la sedimentabilidad y el índice volumétrico del lodo (IVL) de acuerdo con los protocolos establecidos por el Standard Methods (APHA, AWWA, WEF, 1995) [1 1] y los publicados por el IDEAM (disponibles en la página Web de este instituto) [12].

Determinación de los coeficientes cinéticos en el reactor.

Para el diseño futuro de una planta de tratamiento de las aguas residuales del frigorífico "La Frontera Ltda." fue necesario conocer los coeficientes cinéticos propios del agua residual tales como:

K: Tasa máxima de remoción de sustrato / unidad de microorganismo $d^{-1}$

$\mathrm{K}_{\mathrm{s}}$ : Constante de saturación de sustrato.
Y: Coeficiente de producción de crecimiento.

$K_{d}$ : Coeficiente de respiración endógena $d^{-1}$

Dichos valores se obtuvieron mediante la operación de la planta piloto en las diferentes condiciones de tratamiento del experimento, que permitieron obtener los parámetros requeridos para cuantificar los valores de las constantes cinéticas biológicas. Los parámetros que se utilizaron para determinar valores promedios de $\Theta, S_{o^{\prime}}$ S y X son:

$\mathrm{S}_{0}=\mathrm{DBO} \circ \mathrm{DQO}$ inicial

$S=D B O \circ D Q$ final

$\theta=$ Tiempo de retención celular

$X=$ Sólidos suspendidos volátiles.

Estos parámetros se obtuvieron en el laboratorio de aguas de la Universidad Francisco de Paula Santander (U.F.P.S.), de acuerdo con los protocolos establecidos por el Standard Methods (APHA, AWWA, WEF, 1995) [11] y los publicados por el IDEAM (disponibles en la página Web de este instituto) [12]. Obtenidos estos valores se aplicó el Método de Metcalff y Eddy [8] realizando los respectivos cálculos de las constantes.

Estos experimentos se realizaron entre el mes de Agosto de 2004 hasta el mes de Febrero de 2005.

\section{RESULTADOS Y DISCUSIONES}

Cuantificación del agua residual del Frigorífico "La Frontera Ltda."

En la Tabla 3. y Figura 1. se puede apreciar que el mes en el que más se generó agua residual fue mayo (3.93 l/s); igualmente en este mes se presentaron los mayores caudales promedio de agua rumen $(2.256 \mathrm{l} / \mathrm{s})$ y agua sangre $(1.68 \mathrm{l} / \mathrm{s})$. El mes de Agosto fue el de menor producción de agua residual (2.49 $\mathrm{I} / \mathrm{s}$ ) y aunque en este mes el caudal de agua rumen no fue el más bajo, la disminución en el caudal de agua sangre $(0.89 \mathrm{l} / \mathrm{s})$, contribuyó a la disminución 
Tratamiento biológico de aguas residuales de matadero. Caso: Frigorífico La Frontera Ltda., Villa del Rosario, Norte de Santander

notable del caudal, esto gracias a las capacitaciones que se realizaron a los operarios sobre el uso eficiente del agua en el mes de Julio.

Tabla 3. Caudales máximos, promedios y mínimos del agua residual generados en el Frigorífico "La Frontera Ltda."

\begin{tabular}{|c|c|c|c|c|c|c|c|c|c|}
\hline \multirow{2}{*}{ MESES } & \multicolumn{2}{|c|}{ Q máximo (1/s) } & \multicolumn{3}{c|}{ Q Promedio (l/s) } & \multicolumn{3}{|c|}{ Q mínimo (1/s) } \\
\cline { 2 - 10 } & $\mathbf{Q t}$ & AR & AS & Qt & AR & AS & Qt & AR & AS \\
\hline Mayo & 5.270 & 3.382 & 1.888 & 3.93 & 2.256 & 1.68 & 2.929 & 1.519 & 1.34 \\
\hline Julio & 3.77 & 2.42 & 1.583 & 3.155 & 1.85 & 1.302 & 2.67 & 1.55 & 1.183 \\
\hline Agosto & 2.88 & 1.86 & 1.02 & 2.49 & 1.60 & 0.89 & 2.25 & 1.36 & 0.78 \\
\hline Sep-Oct & 3.37 & 2.086 & 1.516 & 3.126 & 1.7945 & 1.336 & 2.960 & 1.630 & 1.233 \\
\hline Diciembre & 3.7 & 2.095 & 1.52 & 3.37 & 1.78 & 1.59 & 3.104 & 1.57 & 1.22 \\
\hline Febrero & 3.767 & 2.342 & 1.748 & 3.65 & 2.24 & 1.409 & 3.535 & 2.019 & 1.277 \\
\hline Marzo & 3.22 & 1.89 & 1.67 & 2.61 & 1.546 & 1.064 & 2.32 & 1.44 & 0.94 \\
\hline PROMEDIO & $\mathbf{3 . 7 1 1}$ & $\mathbf{2 . 2 9}$ & $\mathbf{1 . 5 7}$ & $\mathbf{3 . 1 9 0 1}$ & $\mathbf{1 . 8 8 1 0}$ & $\mathbf{1 . 2 8 0 1}$ & $\mathbf{2 . 8 2 4}$ & $\mathbf{1 . 5 8 6}$ & $\mathbf{1 . 1 2 5 5}$ \\
\hline
\end{tabular}

Figura 1. Caudal promedio durante los meses de monitoreo

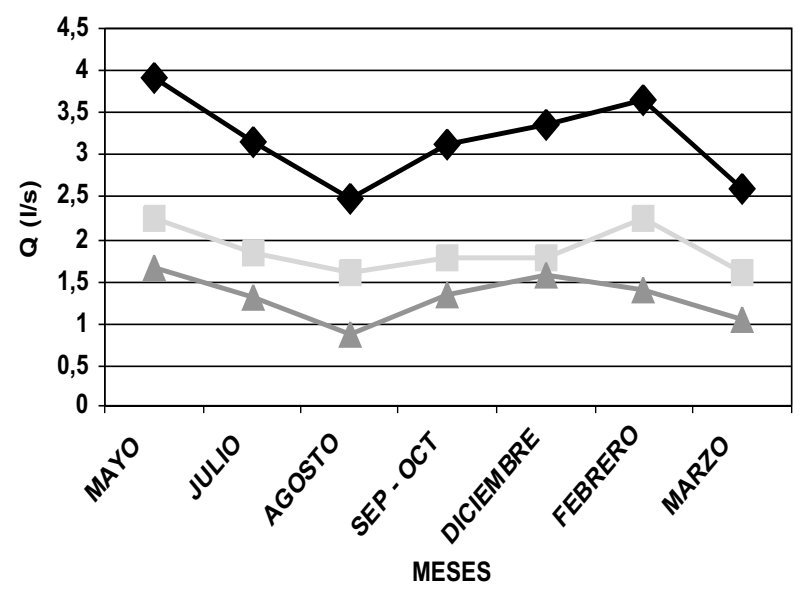

\section{$\neg$ Q. Total $-\mathbf{Q}$. Agua rumen $\quad-\mathbf{Q}$. Agua sangre}

Caudales por etapa de proceso:

El caudal promedio que se registró en las zonas de monitoreo fue: cocina $2.05 \mathrm{l} / \mathrm{s}$, línea de faenado $0.44 \mathrm{l} / \mathrm{s}$ y sala de oreo es de $0.99 \mathrm{l} / \mathrm{s}$.

En la Tabla 4. se observa que en el mes de diciembre se presentaron los mayores caudales en las tres zonas de monitoreo, esto se debió a que en este mes la cantidad de sacrificios fue más grande que los demás meses por ser la temporada alta en el matadero, mientras que agosto fue el mes en donde menos se presentó caudal en las zonas de monitoreo debido a las capacitaciones sobre el uso eficiente del agua.

Tabla 4. Cuantificación del agua residual por etapa de proceso

\begin{tabular}{|c|c|c|c|c|}
\hline $\begin{array}{c}\text { ZONA DE } \\
\text { MONITOREO }\end{array}$ & $\begin{array}{c}\mathrm{Q} \\
\text { Máximo } \\
\mathrm{l} / \mathrm{s}\end{array}$ & $\begin{array}{c}\mathrm{Q} \\
\text { Promedio } \\
\mathrm{l} / \mathrm{s}\end{array}$ & $\begin{array}{c}\mathrm{Q} \\
\text { Mínimo } \\
\mathrm{l} / \mathrm{s}\end{array}$ & $\begin{array}{c}\text { Porcentaje del } \\
\text { caudal total } \\
\text { promedio }\end{array}$ \\
\hline Cocina & 2.32 & 2.05 & 1.69 & $58.90 \%$ \\
\hline Línea de faenado & 0.59 & 0.44 & 0.387 & $12.64 \%$ \\
\hline Sala de oreo & 1.07 & 0.99 & 0.89 & $28.47 \%$ \\
\hline Q. Promedio $(\mathrm{L} / \mathrm{s})$ & 3.98 & 3.48 & 2.96 & $100 \%$ \\
\hline
\end{tabular}

Caracterización del agua residual del Frigorífico "La Frontera Ltda."

Caracterización fisicoquímica. Durante todo el tiempo de estudio se realizaron los análisis respectivos al agua residual para conocer sus características fisicoquímicas y microbiológicas; los resultados se muestran en la Tabla 5.

Tabla 5. Composición físico-química del agua residual del Frigorífico "La Frontera Ltda."

\begin{tabular}{|c|c|c|c|c|}
\hline PARAMETRO & UNIDAD & MÁXIMO & PROMEDIO & MÍNIMO \\
\hline $\mathrm{DQO}$ & $\mathrm{mg} / \mathrm{l} \mathrm{DQO}$ & 16900 & 15232.85 & 14150 \\
\hline $\mathrm{DBO}$ & $\mathrm{mg} / 1 \mathrm{DBO}$ & 7980 & 7547.42 & 6950 \\
\hline Nitratos & $\mathrm{mg} / 1 \mathrm{NO}_{3}$ & 261 & 226.42 & 195.78 \\
\hline Nitritos & $\mathrm{mg} / \mathrm{l} \mathrm{NO}_{2}$ & 4.5 & 3.88 & 3.24 \\
\hline Sulfatos & $\mathrm{mg} / \mathrm{l} \mathrm{SO}_{4}$ & 710 & 657.16 & 615 \\
\hline Fosfatos & $\mathrm{mg} / \mathrm{l} \mathrm{PO}_{4}$ & 276 & 266.16 & 240 \\
\hline Alcalinidad & $\mathrm{mg} / 1 \mathrm{CaCO}_{3}$ & 835 & 801.16 & 780 \\
\hline Acidez & $\mathrm{mg} / 1 \mathrm{CaCO}_{3}$ & 400 & 342.16 & 279 \\
\hline Temperatura & ${ }^{\circ} \mathrm{C}$ & 29 & 28.43 & 26 \\
\hline $\mathrm{pH}$ & $\mathrm{Unidades} \mathrm{de} \mathrm{pH}$ & 7.82 & 7.72 & 7.69 \\
\hline Sólidos Sedimentables & $\mathrm{ml} / 1-\mathrm{h}$ & 760 & 585.86 & 530 \\
\hline Sólidos Totales & $\mathrm{mg} / 1 \mathrm{ST}$ & 6920 & 6689.16 & 6520 \\
\hline Sólidos suspendidos totales & $\mathrm{mg} / 1 \mathrm{SST}$ & 3980.23 & 3386.55 & 2365.48 \\
\hline Sólidos suspendidos volátiles & $\mathrm{mg} / 1 \mathrm{SSV}$ & 2654.12 & 2412.65 & 1980.27 \\
\hline Coliformes fecales & $\mathrm{UFC} / 100 \mathrm{ml}$ & $9^{*} 10^{6}$ & $5^{*} 10^{6}$ & $3 * 10^{6}$ \\
\hline Coliformes Totales & $\mathrm{UFC} / 100 \mathrm{ml}$ & $8^{*} 10^{7}$ & $4^{*} 10^{7}$ & $1 * 10^{7}$ \\
\hline
\end{tabular}

La concentración promedio de DQO fue de $15232,85 \mathrm{mg} / \mathrm{l}$, la mayor concentración se presentó en el mes de junio $(16900 \mathrm{mg} / \mathrm{l})$ y la menor en el mes de octubre (14150 mg/l). Las aguas residuales de matadero se caracterizan por una alta concentración de DQO en sus efluentes [7]; en el caso de 
Tratamiento biológico de aguas residuales de matadero. Caso: Frigorífico La Frontera Ltda., Villa del Rosario, Norte de Santander

Frigorífico "La Frontera Ltda" los valores de las concentraciones de DQO se deben a la gran cantidad de agua sangre que se produce y se encuentra presente en el efluente.

La concentración promedio de DBO obtenida durante el tiempo de estudio es de 7547,42 mg/l. En el mes de diciembre se presentó la mayor concentración de DBO (7980 mg/l), mientras que al igual que en la $D Q O$, en el mes de octubre se registró la menor concentración de DBO en el agua residual (6950 $\mathrm{mg} / \mathrm{l})$. Estos valores se encuentran dentro del rango de los registrados en la literatura para este tipo de aguas [10]

Arranque del sistema de tratamiento de agua

Se realizaron las diferentes conexiones para el arranque de los reactores que se utilizaron para el tratamiento de las aguas residuales. Cada reactor trabajó con inóculos diferentes: el reactor 1 con un inóculo activado a partir del rumen desalojado por el sedimentador, el reactor 2 con un inóculo activado a partir del rumen desalojado por las cribas y el reactor 3 con un inóculo comercial. Cada reactor contenía 2 sopladores de aire que proporcionaban en cada reactor un oxigeno disuelto entre 1.6 a $2.4 \mathrm{mg} /$ I de O.D. El volumen de cada reactor fue de $8 \mathrm{~m}^{3}$, para un total de $24 \mathrm{~m}^{3}$ de agua residual tratada.

Las características del inóculo fueron las siguientes:

Tabla 6. Características del inóculo.

\begin{tabular}{|c|c|c|c|c|}
\hline PARAMETRO & UNIDADES & $\begin{array}{c}\text { INÓCULO } \\
\text { REACTOR 1 }\end{array}$ & $\begin{array}{c}\text { INÓCULO } \\
\text { REACTOR 2 }\end{array}$ & $\begin{array}{c}\text { INÓCULO } \\
\text { REACTOR 3 }\end{array}$ \\
\hline SST & $\mathrm{mg} / \mathrm{l}$ & 4586.32 & 3910 & 2743.35 \\
\hline SSV & $\mathrm{mg} / \mathrm{l}$ & 4333 & 3250 & 2230 \\
\hline SSV/SST & - & 0.95 & 0.83 & 0.81 \\
\hline SEDIMETABILIDAD & $\mathrm{m} / \mathrm{h}$ & 1.178 & 0.98 & 0.78 \\
\hline
\end{tabular}

Para la activación y siembra del lodo se pasó primero agua rumen con un tiempo de retención en el reactor de 2 días, y después se empezó a pasar agua sangre en bajas concentraciones y poco a poco se fue aumentando hasta que se estabilizara el lodo en el reactor. En la Tabla 7 se muestra las concentraciones de agua rumen y agua sangre utilizadas para la activación y siembra del inóculo.

Tabla 7. Concentraciones de agua rumen y agua sangre para la activación del lodo

\begin{tabular}{|c|c|c|c|}
\hline DIA & $\begin{array}{c}\% \\
\text { Agua } \\
\text { rumen }\end{array}$ & $\begin{array}{c}\% \\
\text { Agua } \\
\text { sangre }\end{array}$ & $\begin{array}{c}\text { Tiempo de } \\
\text { aireación } \\
\text { (Días) }\end{array}$ \\
\hline 1 & 100 & 0 & 2 \\
\hline 3 & 90 & 10 & 1 \\
\hline 4 & 80 & 20 & 1 \\
\hline 5 & 65 & 35 & 1 \\
\hline 6 & 50 & 50 & 1 \\
\hline
\end{tabular}

En la Figura 2. se aprecia los reactores con sus respectivas adecuaciones y los sopladores de aire.

Figura 2. Reactores piloto Frigorífico "La Frontera Ltda."

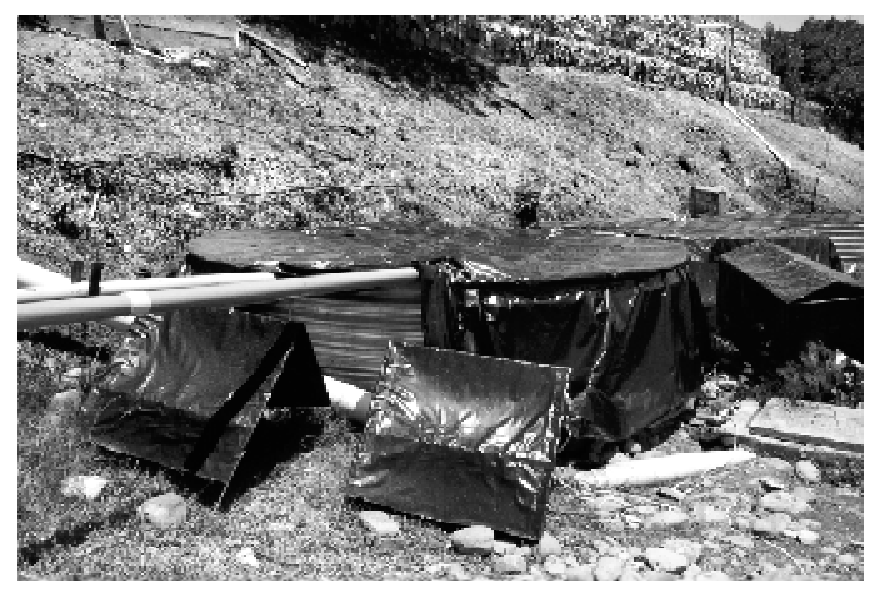

Eficiencia de los reactores de lodos activados.

La mejor remoción en $\mathrm{DBO}$ y $\mathrm{DQO}$, se presentó en el reactor 2 en el experimento 2 bajo las condiciones de un tiempo retención de 36 horas y una concentración de agua rumen del $65 \%$ y $35 \%$ de agua sangre, utilizando como inóculo el rumen separado en las cribas. Estas remociones alcanzadas se deben básicamente al tiempo de retención que fue bastante alto en comparación con el 
Tratamiento biológico de aguas residuales de matadero. Caso: Frigorífico La Frontera Ltda., Villa del Rosario, Norte de Santander

reactor 1 en ese experimento que trabajó con un tiempo de retención de 21 horas.

En el experimento 5 el reactor 1 presentó similares eficiencias pero trabajando con un tiempo de retención de 21 horas y una concentración de agua rumen-agua sangre de 60\% - 40\%; analizando estos resultados este reactor fue mas eficiente que el reactor 2 en el experimento 2, ya que se necesitó de menos tiempo de retención y se trabajó con concentraciones agua sangre más altas para alcanzar similares eficiencias.

En los experimentos donde se trabajaron concentraciones iguales de agua rumen y de agua sangre y tiempos de retención de 21 horas, las mejores eficiencias se presentaron en el reactor 1, en el experimento 6, alcanzando remociones por encima del $60 \%$ tanto en $\mathrm{DQO}$ como en $\mathrm{DBO}$; indicando que el inóculo que mas resiste cambios en la concentración del agua residual es el activado a partir del rumen del sedimentador.

El reactor 3 registró las más bajas remociones tanto en tiempos de retención altos (36 horas) como en tiempos de retención bajos (21 horas), lo que muestra que el inóculo comercial que se utilizó en este reactor no es el apropiado para tratar las aguas del Frigorífico "La Frontera Ltda."

Tabla 8. Comparación de las eficiencias en remoción de $\mathrm{DQO}$ y $\mathrm{DBO}$ en cada reactor por experimento.

\begin{tabular}{|c|c|c|c|c|c|r|}
\hline \multirow{2}{*}{ meses } & \multicolumn{3}{|c|}{ \%Eficiencia DBO } & \multicolumn{3}{c|}{ \%Eficiencia DQO } \\
\cline { 2 - 7 } & R1 & R2 & R3 & R1 & R2 & R3 \\
\hline 1 & 81.45 & - & - & 52.11 & - & - \\
\hline 2 & 82.24 & 81.17 & - & 65.62 & 72.47 & - \\
\hline 3 & 70.75 & 75.13 & 32.46 & 30.89 & 42.15 & 30.4 \\
\hline 4 & 80.97 & 58.39 & 38.4 & 53.03 & 62.33 & 2.41 \\
\hline 5 & 80.28 & 40.12 & - & 70.42 & 60.74 & - \\
\hline 6 & 77.88 & 35 & - & 61.24 & 53.62 & - \\
\hline
\end{tabular}

Donde:

R 1: Reactor 1

R 2: Reactor 2

R 3: Reactor 3

\section{BIOLOGIA DEL REACTOR}

Aislamiento e identificación de microorganismos del proceso de lodos activos.

Se identificaron 4 tipos de microorganismos:

Tabla 9. Microorganismos identificados.

\begin{tabular}{|l|l|}
\hline CEPAS & MICROORGANISMO \\
\hline $1-O_{2}$ & Bacillus cereus \\
\hline $4-6-8-9$ & Bacillus subtilis \\
\hline 1 PAL & Pseudomonas aeruginosa \\
\hline 7 & Flavobacterium sp \\
\hline
\end{tabular}

En los tratamientos biológicos de lodos activos se aprovecha la capacidad colectiva de los microorganismos para degradar una amplia variedad de materiales orgánicos (Stanier et al., 1996), la presencia de los microorganismos Bacillus cereus y Bacillus subtilis es común en este proceso ayudando básicamente a la fijación de fósforo presentes en los fosfatos del agua residual (Madoni y Ghetti, 1979; Antonietti 1982.). En el caso de Pseudumonas aeruginosa son excelentes fijadores de fósforo al igual que los bacillus, pero se han reportado que están involucrados en los procesos de nitrificación de lodos activados. El genero Flavobacterium sp. Fue el único de las cuatro cepas que no se logró identificar su especie. Este género aparece en las últimas etapas de degradación donde el oxigeno se ha consumido en gran parte por ser anaerobios facultativos terminando el proceso de degradación [6]. 
Tratamiento biológico de aguas residuales de matadero. Caso: Frigorífico La Frontera Ltda., Villa del Rosario, Norte de Santander

Identificación de indicadores biológicos. A continuación se presentan los organismos que se identificaron en cada reactor de lodos activados.

Tabla 10. Organismos encontrados en el sistema de lodos activados del Frigorífico "La Frontera Ltda."

\begin{tabular}{|c|c|c|c|}
\hline ORGANISMO & REACTOR 1 & REACTOR 2 & REACTOR 3 \\
\hline Cinetochilium & Presencia baja & No se encontró & No se encontró \\
\hline Stylonichia & Presencia alta & Presencia alta & Presencia baja \\
\hline Oxytrichia & Presencia alta & Presencia media & No se encontró \\
\hline Colpoda & Presencia alta & Presencia baja & No se encontró \\
\hline Epystilis & Presencia alta & Presencia alta & Presencia media \\
\hline Nematodos & Presencia media & Presencia media & Presencia alta \\
\hline Loxophilium & Presencia baja & No se encontró & No se encontró \\
\hline $\begin{array}{c}\text { Dipteros } \\
\text { (psychodidae) }\end{array}$ & No se encontró & Pupas y larvas & Pupas y larvas \\
\hline Vorticella & Presencia alta & Presencia alta & Presencia baja \\
\hline Giridinium & Presencia alta & Presencia alta & Presencia media \\
\hline Phytodinium & Presencia alta & Presencia alta & Presencia baja \\
\hline Sarcodinas (Amibas) & Presencia media & Presencia alta & Presencia alta \\
\hline Quiste de protozoos & Presencia media & Presencia media & Presencia alta \\
\hline Pyxidium & Presencia baja & No se encontró & No se encontró \\
\hline
\end{tabular}

El lodo del reactor 1 fue el que presentó una mayor diversidad de protozoos en comparación con los otros dos reactores, además de reportar una presencia alta de estos organismos en comparación con el lodo del resto de reactores. Lo anterior se explica porque el lodo del reactor 1 se adaptó en óptimas condiciones al agua residual del Frigorífico en comparación con los otros dos reactores.

Descripción de los organismos encontrados.

Phytodinium (Dinoflagelado). El cuerpo de estos individuos es esférico o elipsoidal; sin surcos; los cromatóforos son de forma discoidea, de color castaño amarillento.

Figura 3. Phytodinium (Dinoflagelado)

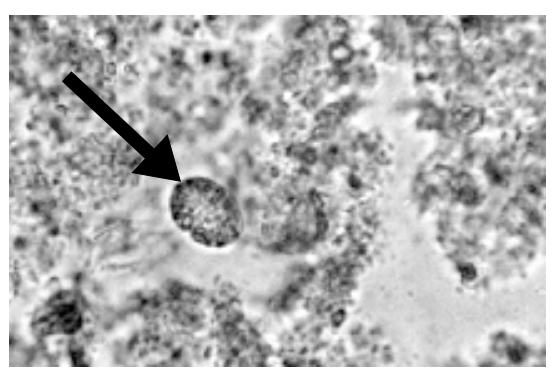

Gyrodinium (Dinoflagelado). Los organismos de este grupo tienen el ánulo descendente en forma de espiral y hacia la izquierda; el sulco se extiende de extremo a extremo; el núcleo es central; poseen púsulas; tiene la superficie lisa o estriada; raramente presentan cromatóforos y su citoplasma es coloreado.

Figura 4. Gyrodinium (Dinoflagelado)

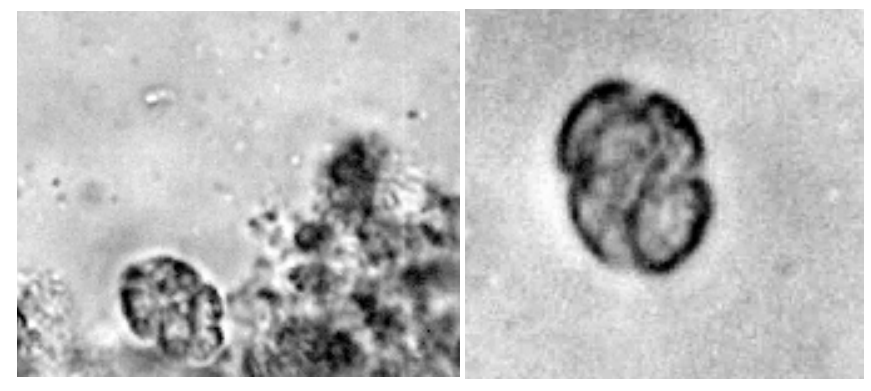

Epistylis (ciliados fijos). El tallo de este grupo de organismos no es contráctil; son solitarios o coloniales. Los organismos de este género tienen forma de campana invertida; generalmente con tallo no dicotómico y no contráctil, formando colonias grandes; se encuentran adheridos a la materia orgánica presente en el medio.

Figura 5. Epistylis (ciliados fijos)

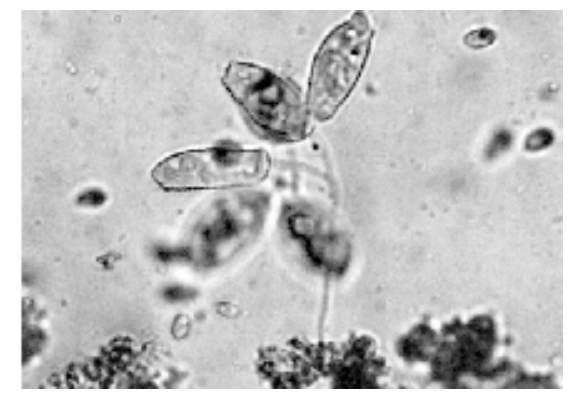

Vorticella. El cuerpo de estos organismos tiene la forma de campana; con tallos contráctiles son sésiles cuando maduros. La forma de campana de estos organismos es invertida; son incoloros, amarillentos o grisáceos; su peristoma se encuentra más o menos extendido exteriormente; la membrana exterior está anillada algunas veces; poseen un tallo contráctil; el 
Tratamiento biológico de aguas residuales de matadero. Caso: Frigorífico La Frontera Ltda., Villa del Rosario, Norte de Santander

macronúcleo tiene forma de banda; tienen un micronúcleo; una o dos vacuolas contráctiles y son solitarios.

Figura 6. Vorticella

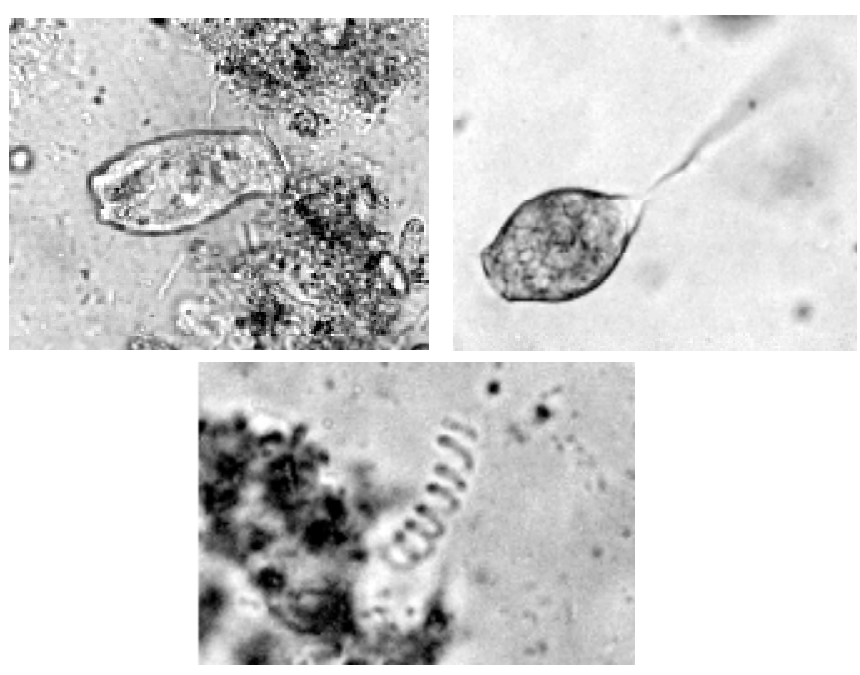

Caracterización de lodos.

En los experimentos 1, 3 y 4 los valores obtenidos en la sedimentabilidad fueron bajos con respecto a los otros experimentos. El IVL revela las características pobres de Sedimentabilidad debido al gran número de protozoos flagelados y una población apreciable de bacterias que catalogan un lodo en etapa de formación. En los experimentos 2 y 5 los valores de sedimentabilidad fueron altos mostrando un lodo fase de madurez de sedimentación rápida, de floculación pobre y de una apariencia casi granular. Ver Tabla 11.

Tabla 11. Análisis y caracterización de sedimentabilidad e índice volumétrico del lodo.

\begin{tabular}{|c|c|c|}
\hline Experimento & Sedimentabilidad $(\mathrm{m} / \mathrm{h})$ & Índice volumétrico del lodo $(\mathrm{mg} / \mathrm{l})$ \\
\hline 1 & 0.732 & 68.71 \\
\hline 2 & 1.178 & 96.99 \\
\hline 3 & 0.6 & 62.40 \\
\hline 4 & 0.62 & 65.42 \\
\hline 5 & 1.178 & 112.60 \\
\hline
\end{tabular}

Determinación de los coeficientes cinéticos en el reactor.

En esta fase del proyecto se estudió la cinética del tratamiento biológico de lodos activados, para determinar los principios por los cuales los microorganismos presentes en los reactores del Frigorífico "La Frontera Ltda." degradan la materia orgánica, obteniéndose la información básica para diseñar reactores biológicos a escala real. Es de resaltar que cada agua residual es diferente y por tanto cada microorganismo se comporta de acuerdo a las condiciones de sustrato, oxígeno y del inóculo de cada sistema. En Colombia este trabajó es uno de los primeros ya que todavía no se reportan estas coeficientes para este tipo de agua. En el siguiente cuadro se presentan los resultados obtenidos en este estudio.

Tabla 12. Coeficientes cinéticos obtenidos del tratamiento de lodos activados del Frigorífico La Frontera Ltda.

\begin{tabular}{|c|c|c|c|c|}
\hline Coeficiente Cinético & Unidades & Máximo & Promedio & Mínimo \\
\cline { 1 - 4 } $\mathbf{Y}$ & $\mathrm{mg} \mathrm{SSV}$ & & & \\
& $\mathrm{mg}$ sustrato & 0.57 & 0.4016 & 0.39 \\
\hline $\mathbf{K}$ & $\mathrm{d}^{-1}$ & 0.7692 & 0.645 & 0.57 \\
\hline $\mathbf{K}_{\mathbf{d}}$ & $\mathrm{d}^{-1}$ & 0.069 & 0.051 & 0.047 \\
\hline $\mathbf{K}_{\mathbf{s}}$ & $\mathrm{mg} / 1$ & 338.009 & 241.86 & 181 \\
\hline$\mu \mathbf{m a x}$ & $\mathrm{d}^{-1}$ & 0.4348 & 0.2951 & 0.223 \\
\hline
\end{tabular}

El comportamiento de los coeficientes cinéticos se registró dentro de los valores descritos por los autores en las diferentes fuentes bibliográficas [9]. En el mes de noviembre se registró el mejor comportamiento de estas constantes en el reactor, debido a que la actividad microbiológica del lodo fue alta. Los SSV en este mes superaron los $4500 \mathrm{mg} / \mathrm{l}$ ya que la edad del lodo en este mes fue la más óptima para la degradación de la materia orgánica existiendo un equilibrio en comunidad microbiana. En los demás meses el comportamiento de los coeficientes fue casi constante a excepción de Ks que si presentaron variaciones considerables. 
Tratamiento biológico de aguas residuales de matadero. Caso: Frigorífico La Frontera Ltda., Villa del Rosario, Norte de Santander

\section{CONCLUSIONES}

La adecuación de la infraestructura presente en el Frigorífico "La Frontera Ltda.", (tanques circulares y sedimentador) permitió la operación de una planta piloto de lodos activos sin recirculación.

El caudal de agua residual promedio generado en el Frigorífico "La Frontera Ltda.", es 3,19 l/s, el cual está compuesto por un $59 \%(1,88 \mathrm{l} / \mathrm{s})$ de agua rumen generada por las actividades realizadas en el cuarto de vísceras blancas y un $41 \%$ $(1,31 \mathrm{l} / \mathrm{s})$ de agua sangre, que se produce en la línea de faenado, la sala de oreo y el cuarto de vísceras rojas.

La composición fisicoquímica del agua residual del Frigorífico La Frontera Ltda., registró altos valores de DQO de 15235,85 mg/l y de DBO de 7547,42 mg/ I, debido a las altas concentraciones de grasa y aceite aportadas por el agua sangre.

Durante la etapa de operación de la planta piloto, el reactor 1 fue el que mejor se comportó alcanzando las mejores remociones de los parámetros medidos: $58.77 \%$ de remoción de DQO y 78 \% de DBO. Esto se debió a que el lodo de este reactor contenía un equilibrio adecuado de la población microbiana, evidenciado por los SSV que registraron un valor por encima de los $4500 \mathrm{mg} / \mathrm{l}$, y la variedad de organismos encontrados en este reactor.

Los experimentos donde mejor se presentaron remociones de la materia orgánica contaminante fueron los experimentos $1,2,4$ y 5 para el reactor $1 ; 1,2$ y 4 para el reactor 2 y en el reactor 3 los dos experimentos realizados no alcanzaron buenas eficiencias. El tipo de lodo utilizado, el tiempo de retención y los cambios de la concentración de agua rumen y agua sangre en cada experimento influyeron en la eficiencia de cada reactor.
El aislamiento de microorganismos del lodo, permitió obtener 4 cepas, presentes en el proceso de degradación la materia orgánica contaminante; Bacillus cereus, Pseudomonas aeruginosa, Bacillus subtilis y Flavobacterium sp.

Los indicadores analizados muestran que el reactor 1 presentó la mayor variedad de organismos y que se ve reflejado en las eficiencias de este reactor.

Los resultados de la determinación de los coeficientes cinéticos revelan que en el mes de noviembre el comportamiento del lodo fue el más óptimo para la degradación de la materia orgánica, en los demás meses de muestreo los cálculos de las constantes revelan un comportamiento constante y condiciones de lodo en crecimiento y declinación.

\section{AGRADECIMIENTOS}

La investigación fue realizada por la Facultad de Ciencias Agrarias y del Ambiente de la Universidad Francisco de Paula Santander (U.F.P.S.), gracias al apoyo Técnico y económico del FRIGORIFICO LA FRONTERA LTDA.

Al Dr. Jacinto Ruiz, Gerente del FRIGORIFICO LA FRONTERA LTDA, el Sr. Francisco Monsalve miembro de la Junta Directiva de la empresa, a la Junta Directiva y al Técnico Juan Ramírez.

A la M.Sc. Mariorie Sánchez por su asesoría en el diagnóstico de la biología del reactor.

A la Microbióloga Claudia Díaz por su apoyo y asesoría para el aislamiento e identificación de microorganismos del reactor de lodo activo.

Al profesor Salomón Herrera Barrera, Docente de la Universidad de Pamplona por su colaboración en la traducción del resumen. 
Tratamiento biológico de aguas residuales de matadero. Caso: Frigorífico La Frontera Ltda., Villa del Rosario, Norte de Santander

\section{BIBLIOGRAFÍA}

[1] ETXEBARRÍA, Javier. Tratamiento de vertidos de matadero. Chile: sn;1998. 82 pp.

[2] HINCAPIÉ, Muñoz Sonia Yaneth. Mataderos municipales: su administración y operación. Santa fe de Bogotá 1997. 300pp.

[3] ROMERO, Rojas. Jairo Alberto. Tratamiento de Aguas Residuales. Editorial Escuela Colombiana de Ingeniería. Bogota 1998. Cap 17,18.19,20,21 y 22. $1210 \mathrm{pp}$.

[4] TAKAHASHI, Sandra Patricia. Evaluación de alternativa de tratamiento de aguas residuales utilizando la tecnología de lodos activados. Universidad del Valle. Facultad de Ingeniería. Ingeniería Ambiental. Cali, 1996. 950 pp.

[5] PABÓN Sandra Liliana y URBINA Suárez Néstor Andrés. Diseño e implementación de un sistema de tratamiento de agua residual para el Frigorífico "La Frontera Ltda.", en Villa del Rosario - Norte de Santander 2004.

[6] PERILLA, Ana Cristina y TORRES, Elvira. Microbiología de sistemas de tratamiento de aguas residuales. Colombia: UNIVALLE, 1984. 330 p.

[7] OROZCO, J. Tratamiento biológico de las aguas residuales. Universidad de Antioquia. Medellín, 1985 $652 \mathrm{pp}$.

[8] METCALFF \& EDDY Ingeniería de aguas residuales. Tratamiento, vertido y reutilización Volumen I y II. Editorial Mc Graw- Hill. España 1995. 235 pp.

[9] METCALF \& EDDY, «lngeniería de Aguas Residuales. Tratamiento, vertido y reutilización», $3^{a}$ edición, Editorial McGraw-Hill., 1998256 pp.
[10] R.S. RAMALHO.»Tratamiento de las Aguas Residuales Ed. Reverte, Barcelona, 1990462 pp.

[11] APHA (1992) Standard Methods for the Examination of Water and Wastewater. 18th ed. American Public Health Association (APHA), American Water Works Association (AWWA), Water Environment Federation (WEF). Washington DC, EEUU. 1085 pp.

[12] IDEAM (Instituto de Hidrología, Metereologia y Estudios Ambientales de Colombia.) www.ideam.gov.co/ 2004.

[13] KUDO Richard, "Protozoología" Compañía Editorial Continental, México 1972. 880pp.

[14] PIZZI Hugo, SANCHEZ José, HUCK Graciela, "Protozoología" Ediciones Eudocor, Cordoba - Argentina, $1999671 \mathrm{pp}$.

Recibido : Septiembre 21 de 2006

Aceptado: Diciembre 10 de 2006 